\title{
APPLICATIONS OF TOPOLOGY TO DNA
}

\author{
ISABEL K. DARCY and DE WITT SUMNERS \\ Department of Mathematics, Florida State University \\ Tallahassee, Florida 32306, U.S.A. \\ E-mail: darcy@math.utexas.edu,sumners@math.fsu.edu
}

The following is an expository article meant to give a simplified introduction to applications of topology to DNA.

0. Some preliminaries. Many biological processes affect topological properties of DNA. Duplex DNA consists of two backbone strands wound about each other in a righthanded helical fashion. Each strand consists of a sugar phosphate backbone with a nitrogenous base attached to each sugar. The four possible bases are adenine (A), guanine $(\mathrm{G})$, cytosine $(\mathrm{C})$, and thymine $(\mathrm{T})$. The two strands are held together by hydrogen bonding between the bases with A always paired with $\mathrm{T}$, and $\mathrm{G}$ always paired with $\mathrm{C}$.

The DNA of most bacteria and viruses is circular. Although human DNA is linear, it is extremely long and tacked down to a protein scaffold at various points on the DNA. This periodic attachment endows human DNA with topological constraints similar to those for circular DNA. These topological constraints can interfere with vital metabolic cellular processes such as replication and transcription. Most mathematicians have, at some point, taken a strip of paper, put an even number of twists in it before taping the ends together, and cut the strip down the middle. The result is two linked strips of paper. This is what occurs when DNA replicates if one thinks of the two edges of the strip as being the sugar phosphate backbones of the two strands of DNA. Hence, enzymes are required to solve these topological entanglement problems which arise through cellular metabolism and replication. In this case topoisomerases, which are enzymes that mediate the passage of one segment of DNA through an enzyme-bridged transient break in the backbone strands of another DNA segment, are responsible for unlinking the DNA. Other enzymes

1991 Mathematics Subject Classification: Primary 57M25; Secondary $92 \mathrm{E} 10$.

Research of the first author is supported by a fellowship from the Program in Mathematics and Molecular Biology at the University of California at Berkeley, which is supported by the National Science Foundation under grant no. DMS-9406348. The government has certain rights to this material. Research of the second author is partially supported by NSF DMS-9403454.

The paper is in final form and no version of it will be published elsewhere. 
called recombinases break two segments of DNA and interchange the ends, resulting in an exchange of genetic information.

$$
\begin{gathered}
\text { crossing change }=\text { DNA strand passage } \\
\text { figure a }
\end{gathered}
$$
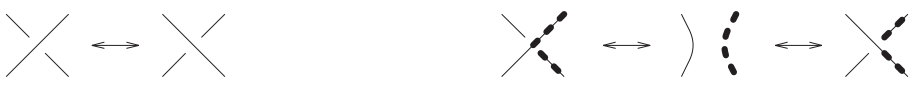

In order to study these enzymes, biologists create unknotted circular DNA in the lab which is then used as the substrate (starting material) for topoisomerase or recombinase experiments. The substrate can be either single-stranded or double-stranded. If it is double-stranded then it can be either nicked or supercoiled. Supercoiled DNA can be modeled with a belt. Take a belt and put an even number of twists in it before closing the belt. Because the belt prefers a specific twist (which is usually zero in the case of a belt), the twist is converted to supercoils. Instead of the two edges of the belt twisting about each other, the centerline of the belt supercoils. Similarly the two strands of DNA prefer a specific twist (about 10.5 base pairs per turn depending upon conditions) and thus over and under twist is converted to supercoils. The DNA is negatively supercoiled if all the crossings of the supercoiled DNA are negative and positively supercoiled if the crossings are all positive.

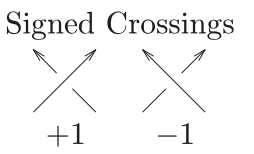

\section{$0, \infty 20$}

negatively supercoiled DNA

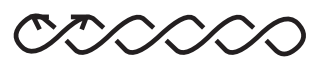

positively supercoiled DNA

Double-stranded DNA is nicked if one of the strands has been cut. Because the DNA is nicked the two strands are free to rotate about each other. Therefore, nicked DNA contains no supercoils.

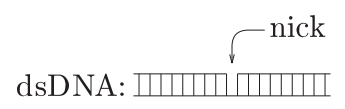

To determine the knot or link type of the product DNA, biologists can take pictures of the DNA (after nicking the DNA to remove supercoils) using an electron microscope (EM). However, this is very difficult, expensive, and ineffective for high crossing number knots and links. Fortunately another method called gel electrophoresis can be used to give much information. The DNA products are put at the top of an agarose gel. Agarose is a sugar polymer which forms a matrix through which the negatively charged DNA can travel when a positive charge is put at the bottom of the gel. The smaller the DNA molecule, the faster it can travel through the gel. For circular DNA molecules which are all the same molecular weight, gel velocity is determined by the average geometric conformation of each molecule as it migrates through the obstruction field imposed by the gel. Knotted DNA travels faster than unknotted DNA, because a knot is more compact than an unknot when tied in a molecule of the same contour length. In some circumstances, gel velocity is determined by the crossing number of the knot [SK] $\mathrm{KB}]$.

The change in knot or link type can be used to study enzyme action as demonstrated in the sections below. The first three sections give very brief introductions. For more 
information, please consult the general references below. The last section describes some newer work with a little more detail.

[KB] V. Katrich, J. Bednar, D. Michoud, R. Scharein, J. Dubochet and A. Stasiak, Geometry and physics of knots, Nature, 384 (1996), 142-145.

[LW] E. Lander and M. Waterman (eds.), Calculating the Secrets of Life, National Academy Press, 1995.

[Le] B. Lewin, Genes V, Oxford University Press, 1994.

[SF] J. Sambrook, E. F. Fritsch and T. Maniatis, Molecular Cloning: A Laboratory Manual, Cold Spring Harbor Laboratory Press, 1989.

[SK] A. Stasiak, V. Katrich, J. Bednar, D. Michoud and J. Dubochet, Electrophoretic mobility of DNA knots, Nature, 384 (1996), 122.

[S] D. Sumners (ed.), New Scientific Applications of Geometry and Topology, Proceedings of Symposia in Applied Math., Vol. 45, AMS, 1992.

[W] M. Waterman (ed.), Mathematical Methods for DNA Sequences, CRC Press, 1989.

1. Differential geometry and DNA. $L k=T w+W r$, a well known formula in differential geometry, may be even better known in molecular biology. The meanings of twist and writhe are probably best demonstrated with a belt. While holding on to one end of the belt, twist the other end $180^{\circ}$ for half a twist, $360^{\circ}$ for 1 full twist, etc. Twist describes how two strands (in this case, the two edges of the belt) wind about each other in space. Now, without letting go of the twist, close up the belt. If you relax the belt, the twist is converted into writhe. If the belt is unknotted, writhe describes the amount of supercoiling. Writhe measures how the center line of the belt winds around in space. It is defined to be the sum of all signed self-crossings of the center line averaged over all projections in $\mathbb{R}^{3}$.

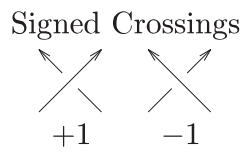

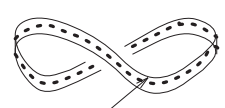

center line

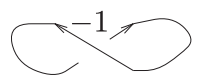

Writhe $=-1$

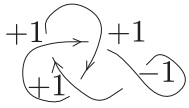

Writhe $=+2$

By moving the belt you are continuously converting writhe to twist and vice versa. Thus, these values are neither integers nor topological invariants. However, their sum, Lk, is both an integer and a topological invariant. The linking number is one-half the sum of all the signed crossings between the two curves. DNA prefers a certain helical twist (10.4 base pairs/turn in the test tube). Therefore changes in linking number are converted to writhe, i.e., the DNA becomes supercoiled. The more supercoiled the DNA is, the more compact it is, and the faster it travels through a gel. Thus the integer differences in linking number can be detected by gel electrophoresis.

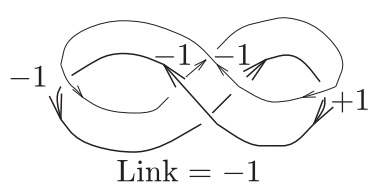

Gel:

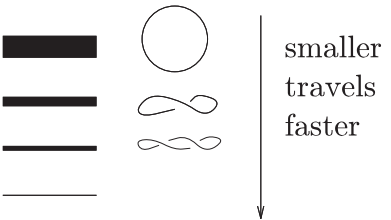

For example, biologists used differences in linking number to determine that a mutant of Gin recombinase was capable of relaxing supercoiled DNA and performing recombina- 
tion using more than one DNA configuration, whereas wild-type Gin can only perform recombination when the DNA is in one specific configuration. This information gave insight into the enzyme mechanism as well. Linking differences have also been used to determine topoisomerase activity.

[W1] J. White, Winding the double helix, in: Calculating the Secrets of Life, National Academy Press, (1995), 153-178.

[W2] J. White, Geomtry and topology of DNA and DNA-protein interactions, in: New Scientific Applications of Geometry and Topology, Proceedings of Symposia in Applied Math., Vol. 45, AMS, (1992), 17-37.

[W3] J. White, An introduction to the geometry and topolgy of DNA structure, in: Mathematical Methods for DNA Sequences, CRC Press, (1989), 225-252.

[KK] A. Klippel, R. Kanaar, R. Kahmann and N. Cozzarelli, Analysis of strand exchange and DNA binding of enhancer-independent Gin recombinase mutants, EMBO 12 (1993), $1047-1057$

2. Graph theory and DNA. Trypanosome is parasite that infects the Tse-tse fly, which in turn infects humans and cows with sleeping sickness. The kinetoplast DNA of trypanosome consists of about 5000 mini circles (small circles of DNA consisting of about 2500 base pairs) and 25 maxi circles (37,000 base pairs) linked together. Biologists wanted to determine how the mini circles were linked together. Electron micrographs of the kinetoplast DNA suggested that the huge kinetoplast link admits a diagram in which each individual minicircle has no self-crossings, and that pairs of minicircles are linked (if at all) like the Hopf link to adjacent minicircles. Ignoring the maxicircles, this assumption allows the complex of minicircles to be translated into graph theory. Each mini circle became a vertex; two vertices were connected by an edge if and only if their respective minicircles were linked, giving rise to a planar graph. For biological reasons, it is believed that the circles are uniformly linked and monolayered. Thus the researchers investigated the seven different ways to tile the plane where all vertices have the same valence and either the tiles have the same shape or there exist two different tile shapes each of which is equally well represented:
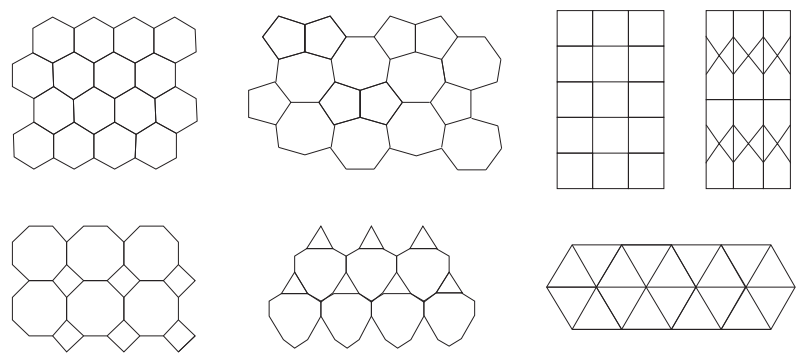

They then looked at the probability that if some circles were randomly broken from the graph that a monomer (single circle not linked to any other circle) or a dimer (two linked circles) or some other configuration would result. For example, if $\mathrm{p}$ is the probability that a circle is broken, then for a 4 -valent graph, the monomer probability is $p^{4}(1-p)$ since the 4 circles that the monomer is linked to would have to be broken 
(probability $=p^{4}$ ), but the monomer itself would have to remain unbroken (probability $=1-p)$. For a 3 -valent graph the monomer probability is $p^{3}(1-p)$.

Biologists determined the above probabilities applied to the trypanosome kDNA by adding endonucleases, enzymes that break the backbone of DNA, in order to randomly break the minicircles. The results were then subjected to gel electrophoresis in order to determine the percentage of monomers versus dimers, etc., that were formed. The experimental results were then compared to the mathematical results to determine that the following is the most likely configuration:

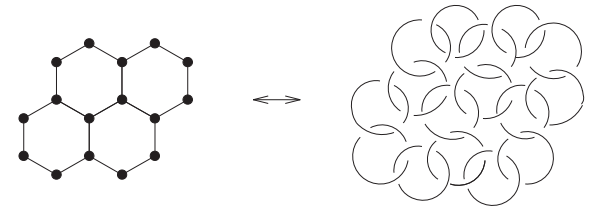

[CR] J. Chen, C. Rauch, J. White, P. Englund and N. Cozzarelli, The topology of the kinetoplast DNA network, Cell 80 (1995) 61-69.

3. Knot theory and DNA: tangles. Some enzymes require DNA to be in a certain configuration in order for the enzyme to act. Electron micrographs of the enzyme-DNA complex show the enzyme as a blob with DNA looping out of it. The configuration of the DNA within the blob cannot be determined from the EM. Thus, the mathematics of tangles has been used in many cases to determine the configuration of the DNA within the enzyme blob.

\begin{abstract}
A 2 string tangle is a 3 -ball with 2 arcs attached to the boundary of the 3-ball.
\end{abstract}

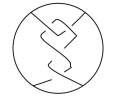

Tangle $\mathrm{T}$
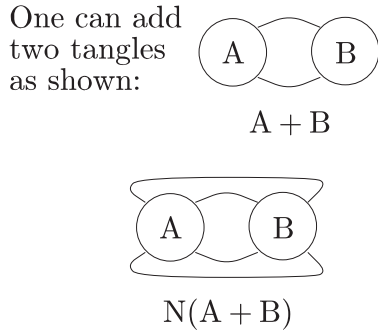

One can also form a knot or link by taking the numerator closure of a tangle or of the sum of tangles:

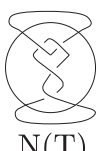

The enzyme-DNA complex can be modeled by writing the DNA as the numerator closure of the sum of three tangles. The tangle $O_{f}$ represents the free DNA, i.e., the part of DNA that is not bound by the enzyme. Tangles $O_{b}$ and $P$ are both bound by the enzyme, but the enzyme only affects the tangle $P$. Tangles $O_{b}$ and $O_{f}$ do not change during the reaction.

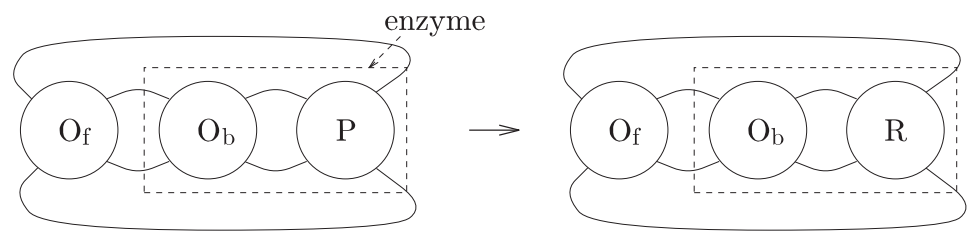

The enzyme action is modeled by replacing tangle $P$ with tangle $R$. Many recombinase enzymes act processively (i.e. it acts more than once in the same place before releasing 
the DNA). This is modeled by adding on multiple $R$ tangles. Since the substrate and products of the experiment are known, this gives several equations which can then be solved for some of the unknown tangles:

$$
\begin{gathered}
\mathrm{N}\left(O_{f}+O_{b}+P\right)=\text { Substrate } \\
\mathrm{N}\left(O_{f}+O_{b}+R\right)=\text { Product } 1 \\
\vdots \\
\mathrm{N}\left(O_{f}+O_{b}+R+\ldots+R\right)=\text { Product } \mathrm{n}
\end{gathered}
$$

[S1] D. W. Sumners, Lifting the curtain, in: Calculating the Secrets of Life, National Academy Press, (1995), 202-235.

[S2] D. W. Sumners, Knot theory and DNA, in: New Scientific Applications of Geometry and Topology, Proceedings of Symposia in Applied Math., Vol. 45, AMS, (1992), 39-72.

[SE] D. W. Sumners, C. Ernst, S. Spengler and N. Cozzarelli, Analysis of the mechanisms of DNA recombination using tangles, Quarterly Review of Biophysics 28 (1995) $253-313$.

4. Knot theory and DNA: unknotting numbers and topoisomerases. Topoisomerases are enzymes that (1.) break the backbone of DNA, (2.) allow passage of another segment of DNA through the transient enzyme-bridged break, and (3.) reseal the break. They are responsible for unknotting, unlinking, and maintaining the proper supercoiling of DNA during the processes of replication, transcription, and recombination. Topoisomerases are also the targets of many antibacterial and anti-cancer drugs [Rc][Wa]. There are two main types of topoisomerases. Type 2 topoisomerases break both backbone strands of double-stranded DNA and thus can change the topology of double-stranded DNA knots and links. Type 1 topoisomerases can only break a single backbone strand of DNA and thus can only change the topology of single-stranded DNA knots and links or double-stranded DNA knots and links if the double-stranded DNA contains a nick (a nick is a broken phosphodiester bond between the sugars of two consecutive bases in one of the strands of dsDNA). Type I topoisomerases can then break the phosphodiester bond between the sugars of the complementary bases, thereby breaking the duplex DNA segment.

Since topoisomerase substrate can be either double-stranded or single-stranded, the line drawings in this paper can represent either double-stranded or single-stranded DNA, depending on context.

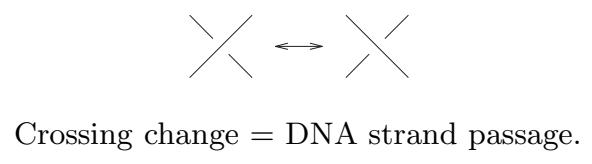

In the test tube, at high concentrations, topoisomerases may produce knots by performing strand passages $[\mathrm{Hs}][\mathrm{DSKC}][\mathrm{WC}][\mathrm{RBW}][\mathrm{Rd}]$. Hence, the unknotting number from knot theory can be used to study topoisomerase action.

Definition. The unknotting number of a knot is the minimum number of crossing changes needed to convert the knot into the unknot where the minimum is taken over all possible diagrams for the knot. 
This is equivalent to determining the minimum number of times needed for topoisomerase to mediate strand passage on a DNA knot to convert it to the unknot, because this temporal sequence of spatial events (strand passages mediated by the enzyme topoisomerase) can be simultaneously visualized in a single knot diagram. To see this, for each strand passage event tie an imaginary string between the segments of string that pass through each other. Simultaneously shorten all the strings until all such pairs are almost touching; now project. Each of the n short strings give rise to a crossing in the resulting diagram; changing all these crossings converts the diagram to a diagram of the unknot. Thus if the unknotting number is $\mathrm{n}$, then biologically, the enzyme must act $\mathrm{n}$ times to convert the knot to the unknot, and mathematically, there exists a diagram of the knot in which one can see $n$ crossings which if changed convert the knot to an unknot.

Example:

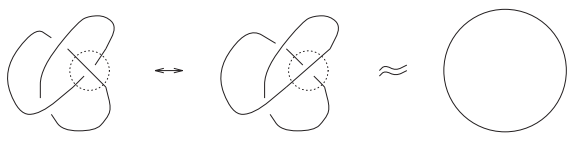

$$
\text { Unknotting number of } 3_{1}=u\left(3_{1}\right)=1
$$

Note that the minimum is taken over all diagrams of the knot.

For example, the minimal diagram of the knot $10_{8}$ requires three crossing changes to change it to the unknot.

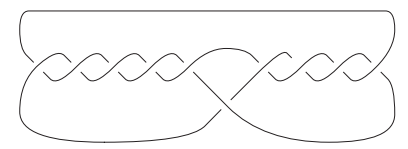

Minimal diagram of the knot $10_{8}$

But Nakanishi in 1983 and Bleiler in 1984 found a more complicated non minimal diagram of 14 crossings of the same knot which required only 2 crossing changes to convert it to the unknot.

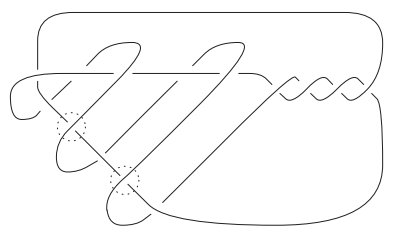

A non-minimal diagram of the knot $10_{8}$ with 14 crossings

Perhaps there exists a diagram of this knot (with possibly a huge number of crossings) which only requires one crossing change to convert it to the unknot. This is the difficult question. Fortunately, there exist bounds on unknotting number ([Ms], $[\mathrm{N} 1])$ :

$$
\begin{gathered}
u(K) \leq \frac{1}{2} \mid \text { number of crossings }-1 \mid \\
u(K) \geq \begin{array}{c}
g^{*}(K) \\
\uparrow \\
4-\text { genus }
\end{array} \quad \begin{array}{c}
{ }^{2}|\sigma(K)| \\
\text { signature }
\end{array}
\end{gathered}
$$

$u(K) \geq s d(K)=$ surgery description number of $K$ 
Many of the above invariants have been calculated and can be found in a knot theory book or at the URL http://www.math.fsu.edu/ ${ }^{\sim}$ darcy.

For example, $\sigma\left(10_{8}\right)=4$ and

$$
u\left(10_{8}\right) \geq \frac{1}{2}\left|\sigma\left(10_{8}\right)\right| \geq \frac{1}{2}(4)=2 .
$$

Thus $u\left(10_{8}\right)=2$. No diagram of this knot can be changed to the unknot with only one crossing change.

The unknotting number can be generalized to a metric on knot types where the distance between any two knots is defined as follows:

Definition (Strand passage metric on knot types):

$u\left(K_{1}, K_{2}\right)=$ minimum number of strand passages needed to convert $K_{1}$ to $K_{2}$ where the minimum is taken over all diagrams.

This satisfies all the properties of a metric:

1.) $u\left(K_{1}, K_{2}\right)=0$ if and only if $K_{1}=K_{2}$.

2.) $u\left(K_{1}, K_{2}\right)=u\left(K_{2}, K_{1}\right)$.

3.) $u\left(K_{1}, K_{2}\right) \leq u\left(K_{1}, K\right)+u\left(K, K_{2}\right)$, for any knot $K$.

Example: $u\left(K_{1}, K_{2}\right) \leq u\left(K_{1}, 0_{1}\right)+u\left(0_{1}, K_{2}\right)$, where $0_{1}$ is the unknot.

$$
\text { I.e., } u\left(K_{1}, K_{2}\right) \leq u\left(K_{1}\right)+u\left(K_{2}\right) \text {. }
$$

That is, the distance between two knots is less than or equal to the sum of their unknotting numbers. However, there may be a shorter path that does not go through the unknot. By doing the crossing changes on a particular diagram, either by hand or by computer, one can easily determine upper bounds for the distance between two given knots. The difficult question is then knowing whether or not there exists a shorter path. For this lower bounds are needed. Murakami[Mk] generalized Murasugi's signature lower bound for the unknotting number to obtain the following lower bound for the strand passage metric:

$$
u\left(K_{1}, K_{2}\right) \geq \frac{1}{2}\left|\sigma\left(K_{1}\right)-\sigma\left(K_{2}\right)\right|
$$

This lower bound also holds for semi-oriented links. Example:

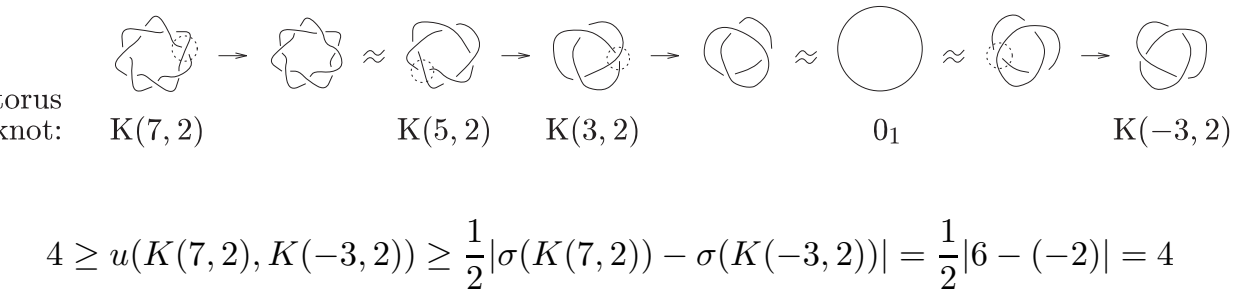

Thus, $u(K(7,2), K(-3,2))=4$.

In general, for $(\mathrm{p}, 2)$ torus knots (p odd):

$$
u(K(p, 2))=\frac{1}{2}(|p|-1)
$$


and

$$
\begin{gathered}
u(K(p, 2), K(q, 2))=\left\{\begin{array}{cl}
\frac{1}{2}|p-q|, & \text { if } \quad p q>0 \\
\frac{1}{2}|p-q|-1, & \text { if } \quad p q<0
\end{array}\right. \\
\text { since } \sigma(K(p, 2))=\left\{\begin{array}{lll}
p-1 & \text { if } & p>0 \\
p+1 & \text { if } & p<0
\end{array}\right.
\end{gathered}
$$

Many of the unknotting number lower bounds such as signature were found by determining the effect a crossing change has on a particular invariant followed by an induction argument.

The strand passage metric table on the next page lists the distance between two knots up to mirror images (since $\left.u\left(K_{1}, K_{2}\right)=u\left(K_{1}^{*}, K_{2}^{*}\right)\right)$. Unfortunately, not all values are known. For example the distance between $4_{1}$ and $5_{1}$ is either 2 or 3 . That is, $4_{1}$ can be changed to $5_{1}$ by only 3 strand passages. However, it is possible that there exists a projection of $4_{1}$ in which only 2 strand passages are required to change $4_{1}$ to $5_{1}$, but there is no way to change $4_{1}$ to $5_{1}$ with only 1 strand passage. Also note that the knot designations refer to those given in Rolfsen's table of knots and not Burde and Zieschang's ([BZ]'s $5_{2}=[\mathrm{R}]$ 's $\left.5_{2}^{*}\right)$.

This metric was calculated for 4-plat knots and composites of 4-plat knots by computer (with 8 crossing non 4-plats put in by hand) using the following information:

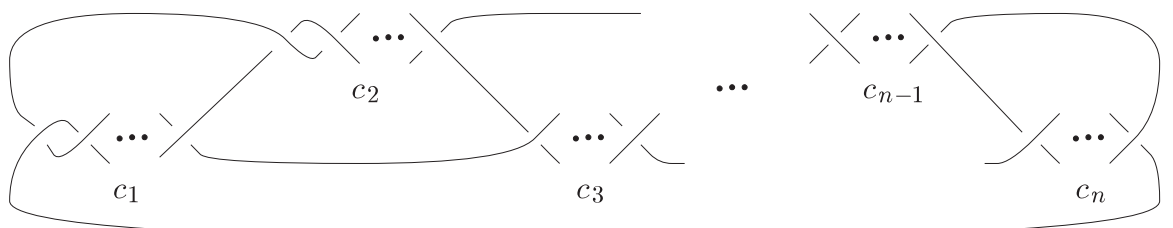

$$
\text { 4-plat }\left\langle c_{1}, \ldots, c_{n}\right\rangle
$$

(1.) $d_{2}\left(\left\langle c_{1}, \ldots, c_{i}, \ldots, c_{n}\right\rangle,\left\langle c_{1}, \ldots, c_{i}-2, \ldots, c_{n}\right\rangle\right)=1$

(2.) $[\mathrm{T}][\mathrm{DS}]$ Classification of distance one 4-plats.

(3.) Triangle Inequality.

(4.) $[\mathrm{Mk}]: d_{2}\left(K_{1}, K_{2}\right) \geq \frac{1}{2}\left|\sigma\left(K_{1}\right)-\sigma\left(K_{2}\right)\right|$.

(5.) $[\mathrm{Mk}]$ Linking form requirements on $\left|H_{1}\left(M_{K}\right)\right|$.

(6.) $[\mathrm{DS}]$ Homology requirements on $H_{1}\left(M_{K}\right)$.

(7.) $[\mathrm{Sc}][\mathrm{Zh}]$ : Unknotting number one knots are prime.

This metric gives the following information about topoisomerase experiments:

1.) The minimum number of times topoisomerase must perform strand passage to inter-convert knots.

2.) All possible reaction pathways in a topoisomerase experiment if all intermediates are known.

For example, in an unpublished experiment of J. Wang, a topoisomerase acting on single-stranded circular DNA produced exclusively $(+)(2 \mathrm{n}+1)$ torus knots which correspond to $(2 n+1)_{1}$ in the knot table. Suppose we are interested in the knot $5_{1}$. The metric 
tells us that (1.) topoisomerase had to act to act at least two times on the unknotted DNA substrate in order to produce the knot $5_{1}$. and (2.) since all products are of the form $(2 n+1)_{1}, 5_{1}$ can only be produced from topoisomerase action on the knot $3_{1}$ or the knot $7_{1}$. This kind of reasoning tells us that the only possible reaction pathway in this experiment is $0_{1} \leftrightarrow 3_{1} \leftrightarrow 5_{1} \leftrightarrow 7_{1} \leftrightarrow \ldots$

\begin{tabular}{|c|c|c|c|c|c|c|c|c|c|c|c|c|}
\hline & $0_{1}$ & $3_{1}$ & $44_{1}$ & $5_{1}$ & $5_{2}$ & $6_{1}$ & $6_{2}$ & $6_{3}$ & $3_{1} \# 3_{1}$ & $3_{1} \# 3_{1}^{*}$ & $7_{1}$ & $7_{2}$ \\
\hline $3_{1}$ & 1 & 0 & 2 & 1 & 1 & 2 & 1 & 1 & 1 & 1 & 2 & 2 \\
\hline $3_{1}^{*}$ & 1 & 2 & 2 & 3 & 2 & 2 & 2 & 1 & 3 & 1 & 4 & 2 \\
\hline $4_{1}$ & 1 & 2 & 0 & $2-3$ & 2 & 1 & 1 & 2 & $2-3$ & $2-3$ & $3-4$ & 2 \\
\hline $5_{1}$ & 2 & 1 & $2-3$ & 0 & 1 & $2-3$ & 2 & 2 & 2 & 2 & 1 & 2 \\
\hline $5_{1}^{*}$ & 2 & 3 & $2-3$ & 4 & 3 & $2-3$ & 3 & 2 & 4 & 2 & 5 & 3 \\
\hline $5_{2}$ & 1 & 1 & 2 & 1 & 0 & 2 & 2 & 2 & 2 & 2 & 2 & 1 \\
\hline $5_{2}^{*}$ & 1 & 2 & 2 & 3 & 2 & 2 & 2 & 2 & 3 & 2 & 4 & 2 \\
\hline $6_{1}$ & 1 & 2 & 1 & $2-3$ & 2 & 0 & 1 & 2 & $2-3$ & $1-3$ & $3-4$ & 2 \\
\hline $6_{1}^{*}$ & 1 & 2 & 1 & $2-3$ & 2 & 1 & 2 & 2 & $2-3$ & $1-3$ & $3-4$ & 2 \\
\hline $6_{2}$ & 1 & 1 & 1 & 2 & 2 & 1 & 0 & 2 & 2 & 2 & $2-3$ & 2 \\
\hline $6_{2}^{*}$ & 1 & 2 & 7 & 3 & 2 & 2 & 2 & 2 & 3 & 2 & 4 & 2 \\
\hline $6_{3}$ & 1 & 1 & 2 & 2 & 2 & 2 & 2 & 0 & 2 & 2 & 3 & 2 \\
\hline $3_{1} \# 3_{1}$ & 2 & 1 & $2-3$ & 2 & 2 & $2-3$ & 2 & 2 & 0 & 2 & $2-3$ & $2-3$ \\
\hline $3_{1}^{*} \# 3_{1}^{*}$ & 2 & 3 & $2-3$ & 4 & 3 & $2-3$ & 3 & 2 & 4 & 2 & 5 & 3 \\
\hline $3_{1} \# 3_{1}^{*}$ & 2 & 1 & $2-3$ & 2 & 2 & $1-3$ & 2 & 2 & 2 & 0 & 3 & $2-3$ \\
\hline $7_{1}$ & 3 & 2 & $3-4$ & 1 & 2 & $3-4$ & $2-3$ & 3 & $2-3$ & 3 & 0 & 2 \\
\hline $77_{1}^{*}$ & 3 & 4 & $3-4$ & 5 & 4 & $3-4$ & 4 & 3 & 5 & 3 & 6 & 4 \\
\hline $7_{2}$ & 1 & 2 & 2 & 2 & 1 & 2 & 2 & 2 & $2-3$ & $2-3$ & 2 & 0 \\
\hline $7_{2}^{*}$ & 1 & 2 & 2 & 3 & 2 & 2 & 2 & 2 & 3 & $2-3$ & 4 & 2 \\
\hline $7_{3}$ & 2 & 3 & $2-3$ & 4 & 3 & $2-3$ & 3 & $2-3$ & 4 & $2-3$ & 5 & 3 \\
\hline $7_{3}^{*}$ & 2 & 2 & $2-3$ & 1 & 1 & $2-3$ & $2-3$ & $2-3$ & $2-3$ & $2-3$ & 1 & 1 \\
\hline $7_{4}$ & 2 & $2-3$ & $2-3$ & $3-4$ & $2-3$ & $2-3$ & $2-3$ & 2 & $3-4$ & 2 & $4-5$ & $2-3$ \\
\hline $7_{4}^{*}$ & 2 & 1 & $2-3$ & 2 & 1 & $2-3$ & 2 & 2 & 2 & 2 & 2 & 2 \\
\hline 75 & 2 & 1 & $2-3$ & 1 & 1 & $2-3$ & 2 & 2 & 2 & 2 & 1 & 1 \\
\hline $77_{5}^{*}$ & 2 & 3 & $2-3$ & 4 & 3 & $2-3$ & 3 & 2 & 4 & 2 & 5 & 3 \\
\hline $7_{6}$ & 1 & 1 & 1 & 2 & 1 & 2 & 2 & 2 & 2 & 2 & $2-3$ & 1 \\
\hline $7_{6}^{*}$ & 1 & 2 & 1 & 3 & 2 & 2 & 2 & 2 & 3 & 2 & 4 & 2 \\
\hline $7_{7}$ & 1 & 2 & 1 & $2-3$ & 2 & 2 & 2 & 2 & $2-3$ & $1-2$ & $3-4$ & 2 \\
\hline $7_{7}^{*}$ & 1 & 1 & 1 & 2 & 2 & 2 & 2 & 2 & 2 & $1-2$ & 3 & 2 \\
\hline $3_{1} \# 4_{1}$ & 2 & 1 & 1 & $1-2$ & $1-2$ & 2 & 2 & 2 & 2 & 2 & $2-3$ & $2-3$ \\
\hline $3_{1}^{*} \# 4_{1}$ & 2 & $2-3$ & 1 & $3-4$ & $2-3$ & 2 & 2 & 2 & $3-4$ & 2 & $4-5$ & $2-3$ \\
\hline $8_{1}$ & 1 & 2 & 2 & $2-3$ & 2 & 1 & 2 & 2 & $2-3$ & $2-3$ & $3-4$ & 2 \\
\hline $8_{1}^{*}$ & 1 & 2 & 2 & $2-3$ & 2 & 2 & 2 & 2 & $2-3$ & $2-3$ & $3-4$ & 2 \\
\hline $8_{2}$ & 2 & 1 & 2 & 1 & 2 & 2 & 1 & 2 & 2 & 2 & 2 & $2-3$ \\
\hline $8_{2}^{*}$ & 2 & 3 & 2 & 4 & 3 & $2-3$ & 3 & 2 & 4 & 2 & 5 & 3 \\
\hline $8_{3}$ & 2 & $2-3$ & 2 & $2-4$ & $2-3$ & 1 & 2 & $2-3$ & $2-4$ & $2-4$ & $3-5$ & $2-3$ \\
\hline 84 & 2 & 2 & 1 & $2-3$ & $2-3$ & 2 & 1 & $2-3$ & $2-3$ & $2-3$ & $2-4$ & $2-3$ \\
\hline $8_{4}^{*}$ & 2 & $2-3$ & 1 & $3-4$ & \begin{tabular}{|l|}
$2-3$ \\
\end{tabular} & 1 & 2 & $2-3$ & $3-4$ & $2-3$ & $4-5$ & $2-3$ \\
\hline $8_{5}$ & 2 & 3 & 2 & 4 & 3 & $2-3$ & 3 & $2-3$ & 4 & $2-3$ & 5 & 3 \\
\hline $8_{5}^{*}$ & 2 & $1-2$ & 2 & $2-3$ & $1-3$ & 2 & 1 & $2-3$ & 1 & $2-3$ & $1-4$ & $1-3$ \\
\hline $8_{6}$ & 2 & 1 & 2 & 2 & 2 & 1 & 1 & 2 & 2 & 2 & $2-3$ & $2-3$ \\
\hline $8_{6}^{*}$ & 2 & $2-3$ & 2 & $3-4$ & $2-3$ & 2 & $2-3$ & 2 & $3-4$ & 2 & $4-5$ & $2-3$ \\
\hline $8_{7}$ & 1 & 2 & 2 & 3 & 2 & 2 & 2 & 1 & 3 & 2 & 4 & 2 \\
\hline
\end{tabular}


[Bl] S. Bleiler, A note on unknotting number, Math. Proc. Camb. Phil. Soc. 96 (1984), 469-471.

[BZ] G. Burde and H. Zieschang, Knots, De Gruyter Studies in Math. 5, Walter de Gruyter, 1985.

[C] J. Conway, An enumeration of knots and links, and some of their algebraic properties, in: Computational Problems in Abstract Algebra, Pergamom Press, 1969, 329358.

[DS] I. Darcy and D. Sumners, A strand passage metric for topoisomerase action, in: Knots '96, World Scientific, 1997, 267-278.

[DSKC] F. Dean, A. Stasiak, T. Koller and N. Cozzarelli, Duplex DNA knots produced by Escherichia coli Topoisomerase I, JBC 260 (1985), 4975-4983.

[Hs] T. Hsieh, Knotting of the Circular Duplex DNA by Type II DNA Topoisomerase form Drosophila melanogaster, JBC 258 (1983), 8413-8420.

[Mk] H. Murakami, Some metrics on classical knots, Math. Ann. 270 (1985), 35-45.

[Ms] K. Murasugi, On a certain numerical invariant of link type, Amer. Math. Soc. 117 (1965), 387-422.

[N1] Y. Nakanishi, Unknotting numbers and knot diagrams with the minimum crossings, Math. Sem. Notes 11 (1983), 257-258.

[N2] Y. Nakanishi, A note on unknotting number, Seminar Notes Kobe Univ. 9 (1981), 99-108.

[Rl] D. Rolfsen, Knots and Links, Publish or Perish, 1990.

[Rc] J. Roca, The mechanisms of DNA topoisomerases, TIBS 20 (1995), 156-160.

[RBW] J. Roca, J. Berger and J. Wang, On the simultaneous Binding of eukaryotic DNA Topoisomerase II to a pair of double-stranded DNA helices, JBC 268 (1993) 1425014255.

[Rd] A. Rodriguez-Campos, DNA knotting abolishes in vitro chromatin assembly, JBC 271 (1996) 14150-14155.

[Sc] M. Scharlemann, Unknotting number one knots are prime, Invent. Math. 82 (1985) $37-55$.

[T] I. Torisu, The determination of the pairs of two-bridge knots or links with Gordian distance one, Proc. Amer. Math. Soc., to appear.

[Wa] J. Wang, DNA topoisomerases, Sci. Am. 247 (1982), 94-109.

[WC] S. Wasserman and N. Cozzarelli, Supercoiled DNA-directed knotting by T4 topoisomerase, J. of Bio. Chem. 266 (1991), 20567-20573.

[Zh] X. Zhang, Unknotting number one knots are prime: a new proof, Proc. AMS 113 (1991), 611-612.

The above four sections are only a very brief sampling of applications of topology to biology. This is truly an extremely rich and fascinating area with questions that mathematicians have been working on without any thoughts about biology as well as questions where mathematicians must work alongside the biologists. 